\title{
Stabilization of Exponentially Unstable Discrete-time Linear Systems by Truncated Predictor Feedbacks*
}

\author{
Yusheng $\mathrm{Wei}^{\dagger}$ and Zongli $\operatorname{Lin}^{\dagger}$
}

\begin{abstract}
Predictor state feedback solves the problem of stabilizing a discrete-time linear system with input delay by predicting the future state with the solution of the state equation and thus rendering the closed-loop system free of delay. The solution of the state equation contains a term that is the convolution of the past control input with the state transition matrix. Thus, the implementation of the resulting predictor state feedback law involves iterative calculation of the control signal. A truncated predictor feedback law results when the convolution term in the state prediction is discarded. When the feedback gain is constructed from the solution of a certain parameterized Lyapunov equation, the truncated predictor feedback law has been shown to achieve asymptotic stabilization of a system that is not exponentially unstable in the presence of an arbitrarily large delay by tuning the value of the parameter small enough. In this paper, we extend this result to exponentially unstable systems. Stability analysis leads to a bound on the delay and a range of the values of the parameter for which the closed-loop system is asymptotically stable as long as the delay is within the bound. The corresponding output feedback result is also derived.

Keywords: Discrete-time, exponentially unstable systems, stabilization, time delay, truncated predictor feedback
\end{abstract}

\section{Introduction}

Time delay in the control input, as a cause of performance deterioration, is ubiquitous in control engineering. A basic control problem for systems with time delay is the problem of stabilization. Thanks to intensive research during the past few decades, various control design methods have been developed and numerous stability conditions established for linear systems with input delay (see, for example, $[2,3,4,5,6,7,15,16,17]$, and [18]). Both continuous-time and discrete-time systems have been studied.

Among the various methods that achieve asymptotic stabilization for a linear system with input delay, predictor state feedback [1] is particularly appealing. It feeds the prediction of the future state into input of the system and results in a closed-loop system free of delay. The state prediction is simply the solution of the state equation of the system. As a result, the predictor feedback consists of two terms, one corresponding to the zero input solution, which is the product of the transition matrix from the current state to the future state in the time equal to the amount of delay, and the other the zero state solution that involves the convolution of the state transition matrix and the past input. The convolution with the past input increases the complexity in the implementation of the feedback law. Discarding the term associated with the zero state solution in the predictor feedback law leads to the so-called truncated predictor feedback (TPF), which leads to remarkable simplification.

Beginning from [13], the TPF has been utilized to stabilize linear systems with input delay. In particular, in [13], it was established that, a continuous-time linear system that is not exponentially unstable can be asymptotically stabilized by the TPF for an arbitrarily large delay if the feedback gain

${ }^{*}$ This work was supported in part by the National Science Foundation under grant CMMI-1462171.

${ }^{\dagger}$ Yusheng Wei and Zongli Lin are with Charles L. Brown Department of Electrical and Computer Engineering, University of Virginia, Charlottesville, VA 22904-4743, USA. Email: yw4dp@virginia.edu, zl5y@virginia.edu 
is constructed by the low gain feedback design technique ([12]) and the value of the low gain parameter is sufficiently small. Following [13], [14] reached a parallel conclusion in the discrete-time setting, that is, a discrete-time linear system that is not exponentially unstable can be stabilized by the low gain based $\mathrm{TPF}$ in the presence of an arbitrarily large delay as long as the low gain parameter is tuned sufficiently small. The low gain feedback designs adopted in [13] and [14] are based on eigenstructure assignment [12]. Alternative TPF designs that adopt a parametric Lyapunov equation based low gain feedback design $[22,23]$ were later proposed in [25] and [24] for continuous-time and discrete-time systems, respectively.

As an extension of the results in [25], [19] develops the truncated predictor feedback for exponentially unstable systems. The feedback law in the TPF law was designed based on the same parametric Lyapunov equation. A bound on the delay that can be tolerated by the TPF law is derived which is inverse proportional to the sum of the unstable poles of the open loop system. The range of the values of the parameter is determined for which the closed-loop system is asymptotically stable as long as the delay is within the bound.

The aim of this paper is to extend the results in [19] to the discrete-time setting. We consider a general discrete-time linear system, which could be exponentially unstable, subject to time-varying delay in the input. A discrete-time parametric Lyapunov equation based approach is adopted to construct feedback gain in the TPF law. Properties of the solution, some of which do not have a continuous-time counterparts, of the parametric Lyapunov equation are established. With the help of these properties, a delay bound and explicit conditions on the feedback gain parameter are then determined under which the asymptotic stability of the system is guaranteed. It is also observed that, as all the exponentially unstable poles of the system approach the unit circle, the delay bound goes to infinity. This observation is consistent with the results in [14] and [24], which showed that for a system whose poles are inside or on the unit circle, asymptotic stabilization can be achieved for an arbitrarily large delay by the TPF. We will also develop output feedback results.

The remainder of this paper is organized as follows. Section 2 states the problem and establishes properties of the solution to the discrete-time parametric Lyapunov equation for exponentially unstable systems. In Section 3, a delay bound and explicit conditions on the parameter are established under which the asymptotic stability of the closed-loop system is guaranteed. Output feedback results are then developed in Section 4. Numerical examples are provided in Section 5. Section 6 concludes the paper.

Notation: Throughout the paper, we use standard notation. In particular, we use $\mathbb{R}, \mathbb{N}$ and $\mathbb{Z}$ to denote the sets of all real numbers, all nonnegative integers and all integers, respectively. Also, for $a, b \in \mathbb{Z}, a \leq b, \mathbf{I}[a, b]$ is the set of all integers in $[a, b]$. For a real matrix $M,\|M\|$ is its induced norm. Finally, $N_{p}$ denotes a neighborhood of a point $p \in \mathbb{R}$.

\section{Problem Statement and Preliminaries}

In this paper, we consider a discrete-time linear system with time-varying input delay,

$$
\left\{\begin{aligned}
x(k+1) & =A x(k)+B u(\phi(k)), \quad k \geq 0, \\
y(k) & =C x(k), \\
x(k) & =\psi(k), k \in \mathbf{I}[-K, 0],
\end{aligned}\right.
$$

where $x(k) \in \mathbb{R}^{n}, u(k) \in \mathbb{R}^{m}$ and $y(k) \in \mathbb{R}^{q}$ are the state vector, input vector and output vector, respectively. The time-varying delay function $\phi(k): \mathbb{N} \rightarrow \mathbb{Z}$ is assumed to have the standard form of $\phi(k)=k-\kappa(k)$, whose inverse function $\phi^{-1}(k): \mathbb{Z} \rightarrow \mathbb{N}$ exists and is known. Also, $\kappa(k) \in \mathbb{N} \rightarrow \mathbb{N}$ denotes time-varying delay that satisfies $\kappa(k) \in \mathbf{I}[0, K]$, where $K \in \mathbb{N} \backslash\{0\}$ is the maximal value of the delay. We note that $\phi^{-1}(k)$ exists as long as $\phi(k)$ is a strictly increasing function of $k$. The initial condition is given by $\psi(k)$ for $k \in \mathbf{I}[-K, 0]$.

It is also assumed that $(A, B)$ is stabilizable and $(A, C)$ is detectable. Without loss of generality, we 
further assume that the pair $(A, B)$ is in the form of

$$
A=\left(\begin{array}{cc}
A_{\mathrm{I}} & 0 \\
0 & A_{\mathrm{O}}
\end{array}\right), B=\left(\begin{array}{c}
B_{\mathrm{I}} \\
B_{\mathrm{O}}
\end{array}\right),
$$

where all eigenvalues of $A_{\mathrm{I}}$ are inside the unit circle and all eigenvalues of $A_{\mathrm{O}}$ are on or outside the unit circle.

Consider the following feedback law for system (1),

$$
u(\phi(k))=F(\gamma) x(k),
$$

where $F(\gamma), \gamma>0$, is a parametric feedback gain matrix which renders $A+B F(\gamma)$ Schur stable. Under the feedback law (3), the closed-loop system is given by

$$
x(k+1)=(A+B F(\gamma)) x(k),
$$

which is asymptotically stable because the matrix $A+B F(\gamma)$ is Schur stable.

Since $\phi^{-1}(k)$ exists and is known, we obtain from $(3)$ that

$$
u(k)=F(\gamma) x\left(\phi^{-1}(k)\right) .
$$

Recall that $\phi(k)=k-\kappa(k)$ and $\kappa(k) \geq 0$ for $k \in \mathbb{N}$, we have $k=\phi\left(\phi^{-1}(k)\right)=\phi^{-1}(k)-\kappa\left(\phi^{-1}(k)\right) \leq$ $\phi^{-1}(k)$. Thus, the right side of (5) contains future state of $x(k)$, namely, $x\left(\phi^{-1}(k)\right)$. This future state can be predicted with the solution of the closed-loop state equation as,

$$
x\left(\phi^{-1}(k)\right)=A^{\phi^{-1}(k)-k} x(k)+\sum_{s=1}^{\phi^{-1}(k)-k} A^{s-1} B u\left(\phi^{-1}(k)-s-\kappa\left(\phi^{-1}(k)-s\right)\right) .
$$

Substitution of (6) in (5) yields the classical predictor state feedback law,

$$
u(k)=F(\gamma) A^{\phi^{-1}(k)-k} x(k)+F(\gamma) \sum_{s=1}^{\phi^{-1}(k)-k} A^{s-1} B u\left(\phi^{-1}(k)-s-\kappa\left(\phi^{-1}(k)-s\right)\right) .
$$

Discarding the term containing the summation sign that involves the past values of the control input results in the truncated predictor feedback law,

$$
u(k)=F(\gamma) A^{\phi^{-1}(k)-k} x(k) .
$$

Following [23], for a controllable pair $(A, B)$ with $A$ being nonsingular, $F(\gamma)$ can be constructed as

$$
F(\gamma)=-\left(I+B^{\mathrm{T}} P(\gamma) B\right)^{-1} B^{\mathrm{T}} P(\gamma) A,
$$

where $P(\gamma)$ is the unique positive definite solution to the discrete-time parametric algebraic Riccati equation

$$
A^{\mathrm{T}} P A-P-A^{\mathrm{T}} P B\left(I+B^{\mathrm{T}} P B\right)^{-1} B^{\mathrm{T}} P A=-\gamma P .
$$

A necessary and sufficient condition for the existence and uniqueness of such a solution $P(\gamma)$ is that

$$
\gamma \in\left(1-|\lambda(A)|_{\min }^{2}, 1\right)
$$

where $|\lambda(A)|_{\text {min }}$ denotes the minimal modulus of all eigenvalues of $A$. We note that $P(\gamma)=W^{-1}(\gamma)$, where $W(\gamma)$ is the unique positive definite solution to the discrete-time Lyapunov equation

which is equivalent to (10).

$$
W(\gamma)-\frac{1}{1-\gamma} A W(\gamma) A^{\mathrm{T}}=-B B^{\mathrm{T}}
$$

The objective of this paper is to establish a bound on the delay and a range of the values of the parameter for which the closed-loop system consisting the system (1) and the truncated predictor feedback law (8) is asymptotically stable as long as the delay is within the bound. The corresponding output feedback result will also be derived. To this end, we need to establish some properties of the solution of the algebraic Riccati equation (10) as well as to generalize an existing version of Jensen's inequality.

The following two lemmas establish properties of $P(\gamma)$ for a general system (1) that may be exponentially unstable. The proofs follow similar steps as in the proof of Proposition 2, Theorem 1 and Corollary 2 in [24], where the systems considered are not exponentially unstable. 
Lemma 1 For a controllable pair $(A, B)$ with $A$ being nonsingular, and any nonnegative integers a and $b$, the following inequality holds for $\gamma \in\left(1-|\lambda(A)|_{\min }^{2}, 1\right)$,

$$
\left(A^{a}\right)^{T} P B\left(I+B^{T} P B\right)^{-1} B^{T}\left(A^{b}\right)^{T} F^{T}\left(I+B^{T} P B\right) F A^{b} B\left(I+B^{T} P B\right)^{-1} B^{T} P A^{a} \leq \omega(a, b) P,
$$

where $P$ is the unique positive definite solution to (10), $F$ is defined in (9), and

$$
\omega(a, b)=\frac{\left(\operatorname{det}^{2}(A)-(1-\gamma)^{n}\right)^{3}}{(1-\gamma)^{(n-1)(a+b+1)}}\left(\operatorname{det}^{2}(A)\right)^{a+b-2} .
$$

Lemma 2 For a controllable pair $(A, B)$ with A being nonsingular, the following inequalities are satisfied for $\gamma \in\left(1-|\lambda(A)|_{\min }^{2}, 1\right)$,

$$
\left\{\begin{aligned}
F^{T}\left(I+B^{T} P B\right) F & \leq \frac{\operatorname{det}^{2}(A)-(1-\gamma)^{n}}{(1-\gamma)^{n-1}} P \\
\left(A^{i}\right)^{T} P A^{i} & \leq\left(\frac{\operatorname{det}^{2}(A)}{(1-\gamma)^{n-1}}\right)^{i} P, \forall i \in \mathbb{N}
\end{aligned}\right.
$$

where $P$ is the unique positive definite solution to (10), and $F$ is defined in (9).

The following lemma generalizes the discrete-time Jensen's Inequality in [9], where matrix $S$ is positive definite.

Lemma 3 For any real positive semi-definite matrix $S$, any integers $n_{1}$ and $n_{2}$ such that $n_{1} \leq n_{2}$, and a vector valued function $x(k): \mathbf{I}\left[n_{1}, n_{2}\right] \rightarrow \mathbb{R}^{n}$,

$$
\left(\sum_{k=n_{1}}^{n_{2}} x(k)\right)^{T} S\left(\sum_{k=n_{1}}^{n_{2}} x(k)\right) \leq\left(n_{2}-n_{1}+1\right) \sum_{k=n_{1}}^{n_{2}} x^{T}(k) S x(k) .
$$

Proof: Let $S=L^{\mathrm{T}} L$, where $L$ is a real matrix of appropriate dimensions. It then follows from the discrete-time Jensen's Inequality [9] that

$$
\begin{aligned}
\left(\sum_{k=n_{1}}^{n_{2}} x(k)\right)^{\mathrm{T}} S\left(\sum_{k=n_{1}}^{n_{2}} x(k)\right) & =\left(\sum_{k=n_{1}}^{n_{2}} x(k)\right)^{\mathrm{T}} L^{\mathrm{T}} L\left(\sum_{k=n_{1}}^{n_{2}} x(k)\right) \\
& =\left(\sum_{k=n_{1}}^{n_{2}} L x(k)\right)^{\mathrm{T}} I\left(\sum_{k=n_{1}}^{n_{2}} L x(k)\right) \\
& \leq\left(n_{2}-n_{1}+1\right) \sum_{k=n_{1}}^{n_{2}}(L x(k))^{\mathrm{T}} I(L x(k)) \\
& =\left(n_{2}-n_{1}+1\right) \sum_{k=n_{1}}^{n_{2}} x^{\mathrm{T}}(k) L^{\mathrm{T}} L x(k) \\
& =\left(n_{2}-n_{1}+1\right) \sum_{k=n_{1}}^{n_{2}} x^{\mathrm{T}}(k) S x(k) .
\end{aligned}
$$

\section{State Feedback Result}

With $(A, B)$ given in (2), system (1) can be written as

$$
\left\{\begin{array}{c}
x_{\mathrm{I}}(k+1)=A_{\mathrm{I}} x_{\mathrm{I}}(k)+B_{\mathrm{I}} u(\phi(k)), \\
x_{\mathrm{O}}(k+1)=A_{\mathrm{O}} x_{\mathrm{O}}(k)+B_{\mathrm{O}} u(\phi(k)),
\end{array}\right.
$$

where $x(k)=\left[\begin{array}{lll}x_{\mathrm{I}}^{\mathrm{T}}(k) & x_{\mathrm{O}}^{\mathrm{T}}(k)\end{array}\right]^{\mathrm{T}}$. Since $A_{\mathrm{I}}$ has only stable eigenvalues, any state feedback law that stabilizes the $x_{\mathrm{O}}$ subsystem would stabilize the entire system. It is thus without loss of generality to make the following assumption, which implies that $(A, B)$ is controllable with $A$ being nonsingular. 
Assumption $1 A$ is exponentially unstable with no eigenvalues inside the unit circle.

Our state feedback result is then stated in the following theorem.

Theorem 1 Let Assumption 1 hold. If $K<\bar{K}$, where $\bar{K}$ is the unique and positive solution to the following equation,

$$
\bar{K}^{2} \beta^{\left(4-\frac{2}{n}\right) \bar{K}}=\frac{2\left(\beta^{\frac{1}{n}}-1\right) \beta}{3\left(\beta-\beta^{-1}\right)^{3}},
$$

where $\beta=\operatorname{det}^{2}(A)$, then, there exists a neighborhood $N_{\gamma^{*}}$ around the point $\gamma^{*}=1-\beta^{-\frac{1}{n}}$ such that for each $\gamma \in N_{\gamma^{*}}$, the truncated predictor feedback law (8) asymptotically stabilizes system (1).

Proof: Under the truncated predictor feedback law (8), the closed-loop system can be written as follows,

$$
\begin{aligned}
x(k+1) & =A x(k)+B u(\phi(k)) \\
& =A x(k)+B F(\gamma) A^{\phi^{-1}(\phi(k))-\phi(k)} x(\phi(k)) \\
& =A x(k)+B F(\gamma) A^{\kappa(k)} x(\phi(k)) \\
& =A_{\mathrm{c}} x(k)+B F(\gamma) \mu(k),
\end{aligned}
$$

where $F(\gamma)$ is defined in $(9), A_{\mathrm{c}}=A+B F(\gamma)$ and $\mu(k)=A^{\kappa(k)} x(\phi(k))-x(k)$. By recursively using $x(k+1)=A x(k)+B F(\gamma) A^{\kappa(k)} x(\phi(k))$, we have

$$
x(k)=A^{\kappa(k)} x(\phi(k))+\sum_{s=1}^{\kappa(k)} A^{s-1} B F(\gamma) A^{\kappa(k-s)} x(k-s-\kappa(k-s)),
$$

which implies that

$$
\mu(k)=-\sum_{s=1}^{\kappa(k)} A^{s-1} B F(\gamma) A^{\kappa(k-s)} x(k-s-\kappa(k-s)) .
$$

We now consider the Lyapunov function $V(x)=x^{\mathrm{T}} P(\gamma) x$, where $P(\gamma)$ is the unique positive definite solution to the algebraic Riccati equation (10). Let $\gamma \in(0,1)$ satisfy (11). Then the forward difference of $V(x(k))$ along the trajectory of the closed-loop system can be evaluated as,

$$
\begin{aligned}
\Delta V & =V(x(k+1))-V(x(k)) \\
& =x^{\mathrm{T}}(k+1) P x(k+1)-x^{\mathrm{T}}(k) P x(k) \\
& =\left(A_{\mathrm{c}} x(k)+B F \mu(k)\right)^{\mathrm{T}} P\left(A_{\mathrm{c}} x(k)+B F \mu(k)\right)-x^{\mathrm{T}}(k) P x(k) \\
& =x^{\mathrm{T}}(k)\left(A_{\mathrm{c}} P A_{\mathrm{c}}-P\right) x(k)+2 x^{\mathrm{T}}(k) A_{\mathrm{c}}^{\mathrm{T}} P B F \mu(k)+\mu^{\mathrm{T}}(k) F^{\mathrm{T}} B^{\mathrm{T}} P B F \mu(k) \\
& =x^{\mathrm{T}}(k)\left(-\gamma P-F^{\mathrm{T}} F\right) x(k)+2 x^{\mathrm{T}}(k) A_{\mathrm{c}}^{\mathrm{T}} P B F \mu(k)+\mu^{\mathrm{T}}(k) F^{\mathrm{T}} B^{\mathrm{T}} P B F \mu(k) \\
& \leq-\gamma x^{\mathrm{T}}(k) P x(k)+\mu^{\mathrm{T}}(k) F^{\mathrm{T}}\left(I+B^{\mathrm{T}} P B\right) F \mu(k),
\end{aligned}
$$

where $A_{\mathrm{c}}^{\mathrm{T}} P A_{\mathrm{c}}-P=-\gamma P-F^{\mathrm{T}} F$ and $F=-B^{\mathrm{T}} P A_{\mathrm{c}}$. Also, for notational brevity, we have suppressed the dependence on $\gamma$ of $P$ and $F$ and will continue to do so in the rest of the paper whenever it does not cause loss of clarity.

Considering that $F^{\mathrm{T}}\left(I+B^{\mathrm{T}} P B\right) F$ is positive semi-definite, and with the help of (14) and Lemma 3, we have

$$
\begin{aligned}
\mu^{\mathrm{T}}(k) F^{\mathrm{T}}\left(I+B^{\mathrm{T}} P B\right) F \mu(k)= & \left(-\sum_{s=1}^{\kappa(k)} A^{s-1} B F A^{\kappa(k-s)} x(k-s-\kappa(k-s))\right)^{\mathrm{T}} F^{\mathrm{T}}\left(I+B^{\mathrm{T}} P B\right) F \\
& \times\left(-\sum_{s=1}^{\kappa(k)} A^{s-1} B F A^{\kappa(k-s)} x(k-s-\kappa(k-s))\right) \\
\leq & \kappa(k) \sum_{s=1}^{\kappa(k)} x^{\mathrm{T}}(k-s-\kappa(k-s))\left(A^{\kappa(k-s)}\right)^{\mathrm{T}} F^{\mathrm{T}} B^{\mathrm{T}}\left(A^{s-1}\right)^{\mathrm{T}} F^{\mathrm{T}}\left(I+B^{\mathrm{T}} P B\right) F
\end{aligned}
$$




$$
\times A^{s-1} B F A^{\kappa(k-s)} x(k-s-\kappa(k-s)),
$$

which, by (9) and Lemma 1 , can be continued as,

$$
\begin{aligned}
\mu^{\mathrm{T}}(k) F^{\mathrm{T}}\left(I+B^{\mathrm{T}} P B\right) F \mu(k) \leq & \kappa(k) \sum_{s=1}^{\kappa(k)} x^{\mathrm{T}}(k-s-\kappa(k-s))\left(A^{\kappa(k-s)+1}\right)^{\mathrm{T}} P B\left(I+B^{\mathrm{T}} P B\right)^{-1} B^{\mathrm{T}}\left(A^{s-1}\right)^{\mathrm{T}} F^{\mathrm{T}} \\
& \times\left(I+B^{\mathrm{T}} P B\right) F A^{s-1} B\left(I+B^{\mathrm{T}} P B\right)^{-1} B^{\mathrm{T}} P A^{\kappa(k-s)+1} x(k-s-\kappa(k-s)) \\
\leq & \kappa(k) \sum_{s=1}^{\kappa(k)} \omega(\kappa(k-s)+1, s-1) x^{\mathrm{T}}(k-s-\kappa(k-s)) P x(k-s-\kappa(k-s)) \\
= & \kappa(k) \sum_{s=1}^{\kappa(k)} \frac{\left(\beta-(1-\gamma)^{n}\right)^{3}}{(1-\gamma)^{(n-1)(\kappa(k-s)+s+1)}} \beta^{\kappa(k-s)+s-2} x^{\mathrm{T}}(k-s-\kappa(k-s)) P \\
& \times x(k-s-\kappa(k-s)) .
\end{aligned}
$$

In view of (15), $\Delta V$ can be further evaluated as,

$$
\begin{aligned}
\Delta V \leq & -\gamma x^{\mathrm{T}}(k) P x(k) \\
& +\kappa(k) \sum_{s=1}^{\kappa(k)} \beta^{\kappa(k-s)+s-2} \frac{\left(\beta-(1-\gamma)^{n}\right)^{3}}{(1-\gamma)^{(n-1)(\kappa(k-s)+s+1)}} x^{\mathrm{T}}(k-s-\kappa(k-s)) P x(k-s-\kappa(k-s)) .
\end{aligned}
$$

Notice here that $s \leq K$ for any $s \in \mathbf{I}[1, \kappa(k)]$, and $\kappa(k-s) \leq K$. It follows that $k-s-\kappa(k-s) \in$ $\mathbf{I}[k-2 K, k]$. Thus, if $V(x(k+z))<\eta V(x(k))$ for any $z \in \mathbf{I}[-2 K, 0]$, where $\eta>1$ is any constant, we have

$$
\begin{aligned}
\Delta V & \leq-\gamma x^{\mathrm{T}}(k) P x(k)+\eta \kappa(k) x^{\mathrm{T}}(k) P x(k) \sum_{s=1}^{\kappa(k)} \beta^{\kappa(k-s)+s-2} \frac{\left(\beta-(1-\gamma)^{n}\right)^{3}}{(1-\gamma)^{(n-1)(\kappa(k-s)+s+1)}} \\
& =-V(x(k))\left(\gamma-\eta \kappa(k) \sum_{s=1}^{\kappa(k)} \beta^{\kappa(k-s)+s-2} \frac{\left(\beta-(1-\gamma)^{n}\right)^{3}}{(1-\gamma)^{(n-1)(\kappa(k-s)+s+1)}}\right) .
\end{aligned}
$$

In order to simplify (16), we note that, Assumption 1 implies that $\beta=\operatorname{det}^{2}(A)>1$. Also note that, $(1-\gamma) \in(0,1)$ for any $\gamma \in(0,1)$, and $\kappa(k-s) \leq K$ for any $s \in \mathbf{I}[1, \kappa(k)]$. It is then clear that $\beta^{\kappa(k-s)} \leq \beta^{K}$ and

$$
\left(\frac{1}{1-\gamma}\right)^{\kappa(k-s)} \leq\left(\frac{1}{1-\gamma}\right)^{K}, \forall s \in \mathbf{I}[1, \kappa(k)] .
$$

Hence, (16) can be continued as,

$$
\begin{aligned}
\Delta V & \leq-V(x(k))\left(\gamma-\eta \kappa(k) \frac{\beta^{K-2}\left(\beta-(1-\gamma)^{n}\right)^{3}}{(1-\gamma)^{(n-1)(K+1)}} \sum_{s=1}^{\kappa(k)}\left(\frac{\beta}{(1-\gamma)^{n-1}}\right)^{s}\right) \\
& \leq-V(x(k))\left(\gamma-\eta \kappa^{2}(k) \frac{\beta^{K-2}\left(\beta-(1-\gamma)^{n}\right)^{3}}{(1-\gamma)^{(n-1)(K+1)}}\left(\frac{\beta}{(1-\gamma)^{n-1}}\right)^{\kappa(k)}\right) \\
& \leq-V(x(k))\left(\gamma-\eta K^{2} \frac{\beta^{2 K-2}\left(\beta-(1-\gamma)^{n}\right)^{3}}{(1-\gamma)^{(n-1)(2 K+1)}}\right) .
\end{aligned}
$$

Now, let $\eta=3 / 2$. Then, if there exists some $\gamma \in(0,1)$ such that

$$
\gamma-\frac{3 K^{2} \beta^{2 K-2}\left(\beta-(1-\gamma)^{n}\right)^{3}}{2(1-\gamma)^{(n-1)(2 K+1)}}>0
$$

then $\Delta V \leq-\zeta(\gamma) V(x(k))$ for some $\zeta(\gamma)>0$, which, by the discrete-time Razumikhin Stability Theorem, implies the asymptotic stability of the closed-loop system.

In what follows, we establish conditions on the delay bound $K$ and the parameter $\gamma$ under which (17) is satisfied. We define a function of $\gamma$ on $\gamma \in(0,1)$ as,

$$
G(\gamma)=\gamma(1-\gamma)^{(n-1)(2 K+1)}-\frac{3}{2} K^{2} \beta^{2 K-2}\left(\beta-(1-\gamma)^{n}\right)^{3}
$$


It is clear that (17) is equivalent to $G(\gamma)>0$. It is also easy to verify that $\gamma=\gamma^{*}$ satisfies (11) and that

$$
G\left(\gamma^{*}\right)=\left(1-\beta^{-\frac{1}{n}}\right) \beta^{-\frac{(n-1)(2 K+1)}{n}}-\frac{3}{2} K^{2} \beta^{2 K-2}\left(\beta-\beta^{-1}\right)^{3}>0 .
$$

Therefore, there exists a neighborhood $N_{\gamma^{*}}$ around the point $\gamma^{*}$ such that for each $\gamma \in N_{\gamma^{*}}, G(\gamma)>0$ is satisfied. To determine the delay bound, we note that (18) is equivalent to

$$
K^{2} \beta^{\left(4-\frac{2}{n}\right) K}<\frac{2 \beta\left(\beta^{\frac{1}{n}}-1\right)}{3\left(\beta-\beta^{-1}\right)^{3}} .
$$

Taking the derivative of the left hand side of (19), we have that, for $\beta>1$ and $K>0$,

$$
\frac{\mathrm{d}}{\mathrm{d} K} K^{2} \beta^{\left(4-\frac{2}{n}\right) K}=\beta^{\left(4-\frac{2}{n}\right) K} K\left(2+K\left(4-\frac{2}{n}\right) \lg \beta\right)>0 .
$$

Since $\left.K^{2} \beta^{\left(4-\frac{2}{n}\right) K}\right|_{K=0}=0$, there exists a unique and positive solution $\bar{K}$ to (13) such that, for each $K \in[1, \bar{K}),(19)$ is satisfied. Therefore, the asymptotic stability of the closed-loop system is guaranteed if $K<\bar{K}$ and $\gamma \in N_{\gamma^{*}}$. This completes the proof.

Remark 1 As all the exponentially unstable eigenvalues of $A$ approach the unit circle, $\operatorname{det}^{2}(A)=\beta \rightarrow$ $1^{+}$. Then the delay bound given in (13) approaches infinity. We can verify this observation by contradiction. Suppose that, as $\beta \rightarrow 1^{+}$, the unique positive solution $\bar{K}$ in (13) is bounded by a finite constant $\widetilde{K}$, namely, $\bar{K} \leq \widetilde{K}$ for each $\beta \in\left(1, \beta^{*}\right]$, for a constant $\beta^{*}>1$. Then, we have

$$
\bar{K}^{2} \beta^{\left(4-\frac{2}{n}\right) \bar{K}} \leq \widetilde{K}^{2}\left(\beta^{*}\right)^{\left(4-\frac{2}{n}\right) \widetilde{K}}<+\infty, \quad \forall \beta \in\left(1, \beta^{*}\right] .
$$

On the other hand, we have

$$
\lim _{\beta \rightarrow 1^{+}} \frac{2 \beta\left(\beta^{\frac{1}{n}}-1\right)}{3\left(\beta-\beta^{-1}\right)^{3}}=\lim _{\beta \rightarrow 1^{+}} \frac{2\left(\beta^{\frac{1}{n}}-1+\frac{1}{n} \beta^{\frac{1}{n}-1}\right)}{9\left(\beta-\beta^{-1}\right)^{2}\left(1+\beta^{-2}\right)}=+\infty,
$$

which contradicts (13). This observation is consistent with the results in [14] and [24], both of which showed that the truncated predictor feedback achieves stabilization for an arbitrarily large delay as long as the open loop system is not exponentially unstable.

\section{Output Feedback Result}

In this section, we consider the problem of stabilizing system (1) by output feedback. We make this following assumption.

Assumption 2 The pair $(A, C)$ is observable.

Given that $(A, C)$ is detectable, Assumption 2 can be made without loss of generality. By putting the system in the observable canonical form, we can readily see that the system output contains only the information of the observable subsystem and an output feedback law that stabilizes the observable subsystem would also stabilize the entire system.

The truncated predictor output feedback law we construct is given by

$$
\left\{\begin{aligned}
\hat{x}(k+1) & =A \hat{x}(k)+B u(\phi(k))+L(C \hat{x}(k)-y(k)), \\
u(k) & =\widetilde{F}(\gamma) A^{\phi^{-1}(k)-k} \hat{x}(k), \\
\hat{x}(k) & =\hat{\psi}(k), k \in \mathbf{I}[-K, 0],
\end{aligned}\right.
$$

where $\hat{x}(k)$ is the estimate of the state $x(k)$, and $L$ is such that $A+L C$ is Schur stable and nonsingular. The existence of such an $L$ is guaranteed by Assumption 2. Also, $\widetilde{F}(\gamma)=-\left(I+B^{\mathrm{T}} \widetilde{P}(\gamma) B\right)^{-1} B^{\mathrm{T}} \widetilde{P}(\gamma) A$, where $\widetilde{P}(\gamma)$ is in the form of

$$
\widetilde{P}(\gamma)=\left(\begin{array}{cc}
0 & 0 \\
0 & P_{\mathrm{O}}(\gamma)
\end{array}\right)
$$

and $P_{\mathrm{O}}(\gamma)$ is the unique positive definite solution to the following parametric algebraic Riccati equation

$$
A_{\mathrm{O}}^{\mathrm{T}} P_{\mathrm{O}} A_{\mathrm{O}}-P_{\mathrm{O}}-A_{\mathrm{O}}^{\mathrm{T}} P_{\mathrm{O}} B_{\mathrm{O}}\left(I+B_{\mathrm{O}}^{\mathrm{T}} P_{\mathrm{O}} B_{\mathrm{O}}\right)^{-1} B_{\mathrm{O}}^{\mathrm{T}} P_{\mathrm{O}} A_{\mathrm{O}}=-\gamma P_{\mathrm{O}}
$$


with $\gamma \in\left(1-\left|\lambda\left(A_{\mathrm{O}}\right)\right|_{\min }^{2}, 1\right)$. The stabilizability of the pair $(A, B)$ guarantees the existence and uniqueness of such a $P_{\mathrm{O}}(\gamma)$.

Let us define $e(k)=x(k)-\hat{x}(k)$. Then we have

$$
e(k+1)=(A+L C) e(k) \text {. }
$$

Thus, the closed-loop system consisting of system (1) and the output feedback law (20) can be written as

$$
\left\{\begin{aligned}
x(k+1) & =A x(k)+B \widetilde{F}(\gamma) A^{\kappa(k)}(x(\phi(k))-e(\phi(k))), \\
e(k+1) & =(A+L C) e(k),
\end{aligned}\right.
$$

which, with $(A, B)$ given in $(2)$ and $P_{\mathrm{O}}(\gamma)$ given in $(21)$, can be expanded as,

$$
\left\{\begin{aligned}
x_{\mathrm{I}}(k+1) & =A_{\mathrm{I}} x_{\mathrm{I}}(k)+B_{\mathrm{I}} F_{\mathrm{O}}(\gamma) A_{\mathrm{O}}^{\kappa(k)}\left(x_{\mathrm{O}}(\phi(k))-e_{\mathrm{O}}(\phi(k))\right), \\
x_{\mathrm{O}}(k+1) & =A_{\mathrm{O}} x_{\mathrm{O}}(k)+B_{\mathrm{O}} F_{\mathrm{O}}(\gamma) A_{\mathrm{O}}^{\kappa(k)}\left(x_{\mathrm{O}}(\phi(k))-e_{\mathrm{O}}(\phi(k))\right), \\
e(k+1) & =(A+L C) e(k),
\end{aligned}\right.
$$

where $x(k)=\left[\begin{array}{ll}x_{\mathrm{I}}^{\mathrm{T}}(k) & x_{\mathrm{O}}^{\mathrm{T}}(k)\end{array}\right]^{\mathrm{T}}, e(k)=\left[\begin{array}{ll}e_{\mathrm{I}}^{\mathrm{T}}(k) & e_{\mathrm{O}}^{\mathrm{T}}(k)\end{array}\right]^{\mathrm{T}}$ and $F_{\mathrm{O}}(\gamma)=-\left(I+B_{\mathrm{O}}^{\mathrm{T}} P_{\mathrm{O}}(\gamma) B_{\mathrm{O}}\right)^{-1} B_{\mathrm{O}}^{\mathrm{T}} P_{\mathrm{O}}(\gamma) A_{\mathrm{O}}$. Since all eigenvalues of $A_{\mathrm{I}}$ are inside the unit circle, the asymptotic stability of the closed-loop system (23) is implied by that of its following subsystem,

$$
\left\{\begin{aligned}
x_{\mathrm{O}}(k+1) & =A_{\mathrm{O}} x_{\mathrm{O}}(k)+B_{\mathrm{O}} F_{\mathrm{O}}(\gamma) A_{\mathrm{O}}^{\kappa(k)}\left(x_{\mathrm{O}}(\phi(k))-e_{\mathrm{O}}(\phi(k))\right), \\
e(k+1) & =(A+L C) e(k) .
\end{aligned}\right.
$$

In what follows, we focus our attention on stability analysis of system (24). It is worth mentioning here that we can no longer assume that all eigenvalues of $A$ are on or outside the unit circle as in Section 3 , since $A+L C$ in (24) is not necessarily block diagonal.

Theorem 2 Let Assumption 2 hold. Then, there exists a sufficiently small constant $\gamma^{*} \in(0,1)$ such that, if $K<\bar{K}$, where $\bar{K}$ is the unique and positive solution to

$$
6 \bar{K}^{2}\left(\frac{\beta_{O}}{\left(1-\gamma^{*}\right)^{n-1}}\right)^{2 \bar{K}}=\frac{\beta_{O}^{2} \gamma^{*}\left(1-\gamma^{*}\right)^{n-1}}{\left(\beta_{O}-\left(1-\gamma^{*}\right)^{n}\right)^{3}},
$$

and $\beta_{O}=\operatorname{det}^{2}\left(A_{O}\right)$, then there exists some $\underline{\gamma} \in\left(0, \gamma^{*}\right)$ such that, for each $\gamma \in\left(\underline{\gamma}, \gamma^{*}\right]$, the truncated predictor output feedback law (20) asymptotically stabilizes system (1).

Proof: Consider the subsystem (24) of the closed-loop system (23). We construct a Lyapunov function as $V\left(x_{\mathrm{O}}(k), e(k)\right)=x_{\mathrm{O}}^{\mathrm{T}}(k) P_{\mathrm{O}}(\gamma) x_{\mathrm{O}}(k)+\rho e^{\mathrm{T}}(k) Q e(k)$, where $\rho$ is a positive constant whose value is to be determined later, and $Q$ is the unique positive definite solution to the Lyapunov equation

$$
(A+L C)^{\mathrm{T}} Q(A+L C)-Q=-I .
$$

Note that the existence and uniqueness of such a $Q$ is guaranteed by the Schur stability of $A+L C$.

Next, we write the first equation in $(24)$ as

$$
x_{\mathrm{O}}(k+1)=A_{\mathrm{OC}} x_{\mathrm{O}}(k)+B_{\mathrm{O}} F_{\mathrm{O}}(\gamma) \lambda_{\mathrm{O}}(k)-B_{\mathrm{O}} F_{\mathrm{O}}(\gamma) A_{\mathrm{O}}^{\kappa(k)} e_{\mathrm{O}}(\phi(k)),
$$

where $A_{\mathrm{OC}}=A_{\mathrm{O}}+B_{\mathrm{O}} F_{\mathrm{O}}(\gamma)$ and $\lambda_{\mathrm{O}}(k)=A_{\mathrm{O}}^{\kappa(k)} x_{\mathrm{O}}(\phi(k))-x_{\mathrm{O}}(k)$. Then, $\lambda_{\mathrm{O}}(k)$ can determined by recursively using the first equation of $(24)$ as,

$$
\lambda_{\mathrm{O}}(k)=-\sum_{s=1}^{\kappa(k)} A_{\mathrm{O}}^{s-1} B_{\mathrm{O}} F_{\mathrm{O}}(\gamma) A_{\mathrm{O}}^{\kappa(k-s)} \hat{x}_{\mathrm{O}}(k-s-\kappa(k-s)),
$$

where $\hat{x}_{\mathrm{O}}(k)=x_{\mathrm{O}}(k)-e_{\mathrm{O}}(k)$.

We now evaluate the forward difference of $V\left(x_{\mathrm{O}}(k), e(k)\right)$ along the trajectory of the closed-loop system (23) as follows,

$$
\begin{aligned}
\Delta V= & V\left(x_{\mathrm{O}}(k+1), e(k+1)\right)-V\left(x_{\mathrm{O}}(k), e(k)\right) \\
= & \left(A_{\mathrm{OC}} x_{\mathrm{O}}(k)+B_{\mathrm{O}} F_{\mathrm{O}} \lambda_{\mathrm{O}}(k)-B_{\mathrm{O}} F_{\mathrm{O}} A_{\mathrm{O}}^{\kappa(k)} e_{\mathrm{O}}(\phi(k))\right)^{\mathrm{T}} P_{\mathrm{O}}\left(A_{\mathrm{OC}} x_{\mathrm{O}}(k)+B_{\mathrm{O}} F_{\mathrm{O}} \lambda_{\mathrm{O}}(k)-B_{\mathrm{O}} F_{\mathrm{O}} A_{\mathrm{O}}^{\kappa(k)} e_{\mathrm{O}}(\phi(k))\right) \\
& +\rho((A+L C) e(k))^{\mathrm{T}} Q(A+L C) e(k)-x_{\mathrm{O}}^{\mathrm{T}}(k) P_{\mathrm{O}} x_{\mathrm{O}}(k)-\rho e^{\mathrm{T}}(k) Q e(k)
\end{aligned}
$$




$$
\begin{aligned}
= & x_{\mathrm{O}}^{\mathrm{T}}(k) A_{\mathrm{O}}^{\mathrm{T}} P_{\mathrm{O}} A_{\mathrm{OC}} x_{\mathrm{O}}(k)+2 x_{\mathrm{O}}^{\mathrm{T}}(k) A_{\mathrm{OC}}^{\mathrm{T}} P_{\mathrm{O}} B_{\mathrm{O}} F_{\mathrm{O}} \lambda_{\mathrm{O}}(k)-2 x_{\mathrm{O}}^{\mathrm{T}}(k) A_{\mathrm{O}}^{\mathrm{T}} P_{\mathrm{O}} B_{\mathrm{O}} F_{\mathrm{O}} A_{\mathrm{O}}^{\kappa(k)} e_{\mathrm{O}}(\phi(k)) \\
& +\lambda_{\mathrm{O}}^{\mathrm{T}}(k) F_{\mathrm{O}}^{\mathrm{T}} B_{\mathrm{O}}^{\mathrm{T}} P_{\mathrm{O}} B_{\mathrm{O}} F_{\mathrm{O}} \lambda_{\mathrm{O}}(k)-2 \lambda_{\mathrm{O}}^{\mathrm{T}}(k) F_{\mathrm{O}}^{\mathrm{T}} B_{\mathrm{O}}^{\mathrm{T}} P_{\mathrm{O}} B_{\mathrm{O}} F_{\mathrm{O}} A_{\mathrm{O}}^{\kappa(k)} e_{\mathrm{O}}(\phi(k)) \\
& +e_{\mathrm{O}}^{\mathrm{T}}(\phi(k))\left(A_{\mathrm{O}}^{\mathrm{T}}\right)^{\kappa(k)} F_{\mathrm{O}}^{\mathrm{T}} B_{\mathrm{O}}^{\mathrm{T}} P_{\mathrm{O}} B_{\mathrm{O}} F_{\mathrm{O}} A_{\mathrm{O}}^{\kappa(k)} e_{\mathrm{O}}(\phi(k))-\rho e^{\mathrm{T}}(k) e(k)-x_{\mathrm{O}}^{\mathrm{T}}(k) P_{\mathrm{O}} x_{\mathrm{O}}(k) \\
\leq & x_{\mathrm{O}}^{\mathrm{T}}(k)\left(-\gamma P_{\mathrm{O}}-F_{\mathrm{O}}^{\mathrm{T}} F_{\mathrm{O}}\right) x_{\mathrm{O}}(k)+\frac{1}{2} x_{\mathrm{O}}^{\mathrm{T}}(k) F_{\mathrm{O}}^{\mathrm{T}} F_{\mathrm{O}} x_{\mathrm{O}}(k)+2 \lambda_{\mathrm{O}}^{\mathrm{T}}(k) F_{\mathrm{O}}^{\mathrm{T}} F_{\mathrm{O}} \lambda_{\mathrm{O}}(k)+\frac{1}{2} x_{\mathrm{O}}^{\mathrm{T}}(k) F_{\mathrm{O}}^{\mathrm{T}} F_{\mathrm{O}} x_{\mathrm{O}}(k) \\
& +2 e_{\mathrm{O}}^{\mathrm{T}}(\phi(k))\left(A_{\mathrm{O}}^{\mathrm{T}}\right)^{\kappa(k)} F_{\mathrm{O}}^{\mathrm{T}} F_{\mathrm{O}} A_{\mathrm{O}}^{\kappa(k)} e_{\mathrm{O}}(\phi(k))+2 \lambda_{\mathrm{O}}^{\mathrm{T}}(k) F_{\mathrm{O}}^{\mathrm{T}} B_{\mathrm{O}}^{\mathrm{T}} P_{\mathrm{O}} B_{\mathrm{O}} F_{\mathrm{O}} \lambda_{\mathrm{O}}(k)-\rho e^{\mathrm{T}}(k) e(k) \\
& +2 e_{\mathrm{O}}^{\mathrm{T}}(\phi(k))\left(A_{\mathrm{O}}^{\mathrm{T}}\right)^{\kappa(k)} F_{\mathrm{O}}^{\mathrm{T}} B_{\mathrm{O}}^{\mathrm{T}} P_{\mathrm{O}} B_{\mathrm{O}} F_{\mathrm{O}} A_{\mathrm{O}}^{\kappa(k)} e_{\mathrm{O}}(\phi(k)) \\
= & -\gamma x_{\mathrm{O}}^{\mathrm{T}}(k) P_{\mathrm{O}} x_{\mathrm{O}}(k)+2 \lambda_{\mathrm{O}}^{\mathrm{T}}(k) F_{\mathrm{O}}^{\mathrm{T}}\left(I+B_{\mathrm{O}}^{\mathrm{T}} P_{\mathrm{O}} B_{\mathrm{O}}\right) F_{\mathrm{O}} \lambda_{\mathrm{O}}(k) \\
& +2 e_{\mathrm{O}}^{\mathrm{T}}(\phi(k))\left(A_{\mathrm{O}}^{\mathrm{T}}\right)^{\kappa(k)} F_{\mathrm{O}}^{\mathrm{T}}\left(I+B_{\mathrm{O}}^{\mathrm{T}} P_{\mathrm{O}} B_{\mathrm{O}}\right) F_{\mathrm{O}} A_{\mathrm{O}}^{\kappa(k)} e_{\mathrm{O}}(\phi(k))-\rho e^{\mathrm{T}}(k) e(k),
\end{aligned}
$$

where $A_{\mathrm{OC}}^{\mathrm{T}} P_{\mathrm{O}} A_{\mathrm{OC}}-P_{\mathrm{O}}=-\gamma P_{\mathrm{O}}-F_{\mathrm{O}}^{\mathrm{T}} F_{\mathrm{O}}$ and $F_{\mathrm{O}}=-B_{\mathrm{O}}^{\mathrm{T}} P_{\mathrm{O}} A_{\mathrm{OC}}$.

In view of (28), Lemma 1 and Lemma 3 , we have

$$
\begin{aligned}
\lambda_{\mathrm{O}}^{\mathrm{T}}(k) F_{\mathrm{O}}^{\mathrm{T}}\left(I+B_{\mathrm{O}}^{\mathrm{T}} P_{\mathrm{O}} B_{\mathrm{O}}\right) F_{\mathrm{O}} \lambda_{\mathrm{O}}(k)= & \left(\sum_{s=1}^{\kappa(k)} \hat{x}_{\mathrm{O}}^{\mathrm{T}}(k-s-\kappa(k-s))\left(A_{\mathrm{O}}^{\mathrm{T}}\right)^{\kappa(k-s)} F_{\mathrm{O}}^{\mathrm{T}} B_{\mathrm{O}}^{\mathrm{T}}\left(A_{\mathrm{O}}^{\mathrm{T}}\right)^{s-1}\right) \\
& \times F_{\mathrm{O}}^{\mathrm{T}}\left(I+B_{\mathrm{O}}^{\mathrm{T}} P_{\mathrm{O}} B_{\mathrm{O}}\right) F_{\mathrm{O}}\left(\sum_{s=1}^{\kappa(k)} A_{\mathrm{O}}^{s-1} B_{\mathrm{O}} F_{\mathrm{O}} A_{\mathrm{O}}^{\kappa(k-s)} \hat{x}_{\mathrm{O}}(k-s-\kappa(k-s))\right) \\
\leq & \kappa(k) \sum_{s=1}^{\kappa(k)} \frac{\left(\beta_{\mathrm{O}}-(1-\gamma)^{n}\right)^{3}}{(1-\gamma)^{(n-1)(\kappa(k-s)+s+1)}} \beta_{\mathrm{O}}^{\kappa(k-s)+s-2} \\
& \times \hat{x}_{\mathrm{O}}^{\mathrm{T}}(k-s-\kappa(k-s)) P_{\mathrm{O}} \hat{x}_{\mathrm{O}}(k-s-\kappa(k-s)) .
\end{aligned}
$$

On the other hand, by Lemma 2 , we have

$$
\left(A_{\mathrm{O}}^{\mathrm{T}}\right)^{\kappa(k)} F_{\mathrm{O}}^{\mathrm{T}}\left(I+B_{\mathrm{O}}^{\mathrm{T}} P_{\mathrm{O}} B_{\mathrm{O}}\right) F_{\mathrm{O}} A_{\mathrm{O}}^{\kappa(k)} \leq\left(1-\frac{(1-\gamma)^{n}}{\beta_{\mathrm{O}}}\right)\left(\frac{\beta_{\mathrm{O}}}{(1-\gamma)^{n-1}}\right)^{\kappa(k)+1} P_{\mathrm{O}}
$$

Then, the evaluation of $\Delta V$ in (29) can be continued as,

$$
\begin{aligned}
\Delta V \leq & -\gamma x_{\mathrm{O}}^{\mathrm{T}}(k) P_{\mathrm{O}} x_{\mathrm{O}}(k)+2 \kappa(k) \sum_{s=1}^{\kappa(k)} \frac{\left(\beta_{\mathrm{O}}-(1-\gamma)^{n}\right)^{3}}{(1-\gamma)^{(n-1)(\kappa(k-s)+s+1)}} \beta_{\mathrm{O}}^{\kappa(k-s)+s-2} \hat{x}_{\mathrm{O}}^{\mathrm{T}}(k-s-\kappa(k-s)) P_{\mathrm{O}} \\
& \times \hat{x}_{\mathrm{O}}(k-s-\kappa(k-s))+2\left(1-\frac{(1-\gamma)^{n}}{\beta_{\mathrm{O}}}\right)\left(\frac{\beta_{\mathrm{O}}}{(1-\gamma)^{n-1}}\right)^{\kappa(k)+1} e_{\mathrm{O}}^{\mathrm{T}}(\phi(k)) P_{\mathrm{O}} e_{\mathrm{O}}(\phi(k)) \\
& -\rho e^{\mathrm{T}}(k) e(k),
\end{aligned}
$$

which, in view of $\hat{x}_{\mathrm{O}}(k)=x_{\mathrm{O}}(k)-e_{\mathrm{O}}(k)$ and $(21)$, can be further continued as,

$$
\begin{aligned}
\Delta V \leq & -\gamma x_{\mathrm{O}}^{\mathrm{T}}(k) P_{\mathrm{O}} x_{\mathrm{O}}(k)+4 \kappa(k) \sum_{s=1}^{\kappa(k)} \frac{\left(\beta_{\mathrm{O}}-(1-\gamma)^{n}\right)^{3}}{(1-\gamma)^{(n-1)(\kappa(k-s)+s+1)}} \beta_{\mathrm{O}}^{\kappa(k-s)+s-2}\left(x_{\mathrm{O}}^{\mathrm{T}}(k-s-\kappa(k-s)) P_{\mathrm{O}}\right. \\
& \left.\times x_{\mathrm{O}}(k-s-\kappa(k-s))+e^{\mathrm{T}}(k-s-\kappa(k-s)) \widetilde{P} e(k-s-\kappa(k-s))\right)-\rho e^{\mathrm{T}}(k) e(k) \\
& +2\left(1-\frac{(1-\gamma)^{n}}{\beta_{\mathrm{O}}}\right)\left(\frac{\beta_{\mathrm{O}}}{(1-\gamma)^{n-1}}\right)^{\kappa(k)+1} e^{\mathrm{T}}(\phi(k)) \widetilde{P} e(\phi(k)) .
\end{aligned}
$$

Since $A+L C$ is nonsingular, we obtain from (22) that

$$
e(\phi(k))=(A+L C)^{-\kappa(k)} e(k) .
$$

Thus, it follows from (33) that

$$
\begin{aligned}
\Delta V \leq & -\gamma x_{\mathrm{O}}^{\mathrm{T}}(k) P_{\mathrm{O}} x_{\mathrm{O}}(k)+4 \kappa(k) \sum_{s=1}^{\kappa(k)} \frac{\left(\beta_{\mathrm{O}}-(1-\gamma)^{n}\right)^{3}}{(1-\gamma)^{(n-1)(\kappa(k-s)+s+1)}} \beta_{\mathrm{O}}^{\kappa(k-s)+s-2} \\
& \times\left(x_{\mathrm{O}}^{\mathrm{T}}\left(k-s-\kappa(k-s) P_{\mathrm{O}} x_{\mathrm{O}}(k-s-\kappa(k-s))+e^{\mathrm{T}}(k-s-\kappa(k-s)) \widetilde{P} e(k-s-\kappa(k-s))\right)\right.
\end{aligned}
$$




$$
+e^{\mathrm{T}}(k)\left(2\left(1-\frac{(1-\gamma)^{n}}{\beta_{\mathrm{O}}}\right)\left(\frac{\beta_{\mathrm{O}}}{(1-\gamma)^{n-1}}\right)^{\kappa(k)+1}\left((A+L C)^{\mathrm{T}}\right)^{-\kappa(k)} \widetilde{P}(A+L C)^{-\kappa(k)}-\rho I\right) e(k) .
$$

In order to simplify (34), we next show that there exist a sufficiently small $\gamma^{*}>0$, such that, for each $\gamma \in\left(0, \gamma^{*}\right]$, there is a sufficiently large $\rho^{*}(\gamma)>0$ such that for each $\gamma \in\left(0, \gamma^{*}\right]$ and each $\rho \in\left[\rho^{*}(\gamma),+\infty\right)$,

$$
\left\{\begin{array}{l}
2\left(1-\frac{(1-\gamma)^{n}}{\beta_{\mathrm{O}}}\right)\left(\frac{\beta_{\mathrm{O}}}{(1-\gamma)^{n-1}}\right)^{\kappa(k)+1}\left((A+L C)^{\mathrm{T}}\right)^{-\kappa(k)} \widetilde{P}(A+L C)^{-\kappa(k)}-\rho I \leq-\gamma \rho Q, \\
\widetilde{P} \leq \rho Q .
\end{array}\right.
$$

Note that the first inequality in (35) is equivalent to

$$
\gamma Q+\frac{2}{\rho}\left(1-\frac{(1-\gamma)^{n}}{\beta_{\mathrm{O}}}\right)\left(\frac{\beta_{\mathrm{O}}}{(1-\gamma)^{n-1}}\right)^{\kappa(k)+1}\left((A+L C)^{\mathrm{T}}\right)^{-\kappa(k)} \widetilde{P}(A+L C)^{-\kappa(k)} \leq I, \forall \kappa(k) \in \mathbf{I}[0, K] .
$$

Let $\gamma^{*} \in\left(1-\left|\lambda\left(A_{\mathrm{O}}\right)\right|_{\min }^{2}, 1\right)$ be such that $\gamma^{*} Q<I$. Then, for any $\gamma \in\left(0, \gamma^{*}\right]$, there exists a $\rho^{*}(\gamma)>0$ such that for all $\gamma \in\left(0, \gamma^{*}\right]$ and $\rho \in\left[\rho^{*}(\gamma),+\infty\right),(35)$ holds.

Thus, for each $\gamma \in\left(0, \gamma^{*}\right]$ and $\rho \in\left[\rho^{*}(\gamma),+\infty\right)$, (34) simplifies to

$$
\begin{aligned}
\Delta V \leq & -\gamma V\left(x_{\mathrm{O}}(k), e(k)\right) \\
& +4 \kappa(k) \sum_{s=1}^{\kappa(k)} \frac{\left(\beta_{\mathrm{O}}-(1-\gamma)^{n}\right)^{3}}{(1-\gamma)^{(n-1)(\kappa(k-s)+s+1)}} \beta_{\mathrm{O}}^{\kappa(k-s)+s-2} V\left(x_{\mathrm{O}}(k-s-\kappa(k-s)), e(k-s-\kappa(k-s))\right) .
\end{aligned}
$$

Now, if $V\left(x_{\mathrm{O}}(k+z), e(k+z)\right)<\eta V\left(x_{\mathrm{O}}(k), e(k)\right), \eta=3 / 2>1$, for each $z \in \mathbf{I}[-2 K, 0]$, then,

$$
\begin{aligned}
\Delta V & \leq\left(-\gamma+4 \eta \kappa(k) \sum_{s=1}^{\kappa(k)} \frac{\left(\beta_{\mathrm{O}}-(1-\gamma)^{n}\right)^{3}}{(1-\gamma)^{(n-1)(\kappa(k-s)+s+1)}} \beta_{\mathrm{O}}^{\kappa(k-s)+s-2}\right) V\left(x_{\mathrm{O}}(k), e(k)\right) \\
& \leq\left(-\gamma+6 K^{2} \frac{\left(\beta_{\mathrm{O}}-(1-\gamma)^{n}\right)^{3}}{(1-\gamma)^{(n-1)(2 K+1)}} \beta_{\mathrm{O}}^{2 K-2}\right) V\left(x_{\mathrm{O}}(k), e(k)\right) .
\end{aligned}
$$

Let

$$
H(\gamma)=-\gamma+6 K^{2} \frac{\left(\beta_{\mathrm{O}}-(1-\gamma)^{n}\right)^{3}}{(1-\gamma)^{(n-1)(2 K+1)}} \beta_{\mathrm{O}}^{2 K-2}, \gamma \in\left(1-\left|\lambda\left(A_{\mathrm{O}}\right)\right|_{\min }^{2}, 1\right)
$$

Then, if there exists a $\gamma \in\left(0, \gamma^{*}\right]$ such that $H(\gamma)<0,(37)$ would imply, by the discrete-time Razumikhin Stability Theorem, that the closed-loop system is asymptotically stable.

Hence, we remain to determine an $\bar{K}>0$ and a range of $\gamma$ such that $H(\gamma)<0$ holds for all $K \leq \bar{K}$ as long as $\gamma$ belongs to the range. To this end, we observe that, if $H\left(\gamma^{*}\right)<0$, then there exists an $\underline{\gamma} \in\left(0, \gamma^{*}\right)$ such that $H(\gamma)<0$ for all $\gamma \in\left(\underline{\gamma}, \gamma^{*}\right]$. Since $H(0)=6 K^{2}\left(\beta_{\mathrm{O}}-1\right)^{3} \beta_{\mathrm{O}}^{2 K-1}>0, \underline{\gamma}>0$.

Note that $H\left(\gamma^{*}\right)<0$ is equivalent to

$$
6 K^{2}\left(\frac{\beta_{\mathrm{O}}}{\left(1-\gamma^{*}\right)^{n-1}}\right)^{2 K}<\frac{\beta_{\mathrm{O}}^{2} \gamma^{*}\left(1-\gamma^{*}\right)^{n-1}}{\left(\beta_{\mathrm{O}}-\left(1-\gamma^{*}\right)^{n}\right)^{3}}
$$

From the left-hand side of (38), we obtain

$$
\left\{\begin{aligned}
& \frac{\mathrm{d}}{\mathrm{d} K}\left(6 K^{2}\left(\frac{\beta_{\mathrm{O}}}{\left(1-\gamma^{*}\right)^{n-1}}\right)^{2 K}\right)>0, \forall K>0 \\
&\left.6 K^{2}\left(\frac{\beta_{\mathrm{O}}}{\left(1-\gamma^{*}\right)^{n-1}}\right)^{2 K}\right|_{K=0}=0
\end{aligned}\right.
$$

from which we conclude that for any $K<\bar{K}$, where $\bar{K}$ is the unique and positive solution to (25), (38) is satisfied. This completes the proof.

Remark 2 It can be readily observed from (25) that the delay bound of the closed-loop system not only depends on $\beta_{O}$, but also on $\gamma^{*}$, which is a positive constant determined by $Q$, as seen in the proof of Theorem 2. Since there is no direct relationship between $\beta_{O}$ and $Q$, it is no longer guaranteed that the delay bound goes to infinity as all the eigenvalues of $A_{O}$ approach the unit circle, namely, $\beta_{O} \rightarrow 1^{+}$. 
However, under some additional conditions on the function $\gamma^{*}\left(\beta_{O}\right)$, the delay bound in (25) does approach infinity as $\beta_{O} \rightarrow 1^{+}$.

In what follows, we first establish a theorem that provides conditions on $\gamma^{*}\left(\beta_{\mathrm{O}}\right)$ under which the delay bound goes to infinity as $\beta_{\mathrm{O}} \rightarrow 1^{+}$. We will then construct two examples to validate this theorem.

Theorem 3 As all eigenvalues of $A_{O}$ approach the unit circle, if there exist a positive constant $\beta_{O}^{*}>1$, an observer gain matrix $L$ and a continuous function $\gamma^{*}\left(\beta_{O}\right)$ on $\beta_{O} \in\left(1, \beta_{O}^{*}\right]$ such that

$$
\begin{aligned}
\gamma^{*}\left(\beta_{O}\right) Q & <I, \forall \beta_{O} \in\left(1, \beta_{O}^{*}\right], \\
\lim _{\beta_{O} \rightarrow 1^{+}} \gamma^{*}\left(\beta_{O}\right) & =0, \\
\lim _{\beta_{O} \rightarrow 1^{+}} \frac{\left(\beta_{O}-1\right)^{3}}{\gamma^{*}\left(\beta_{O}\right)} & =0,
\end{aligned}
$$

where $(A+L C)^{T} Q(A+L C)-Q=-I$, then the delay bound $\bar{K}$ in (25) approaches infinity, namely, $\lim _{\beta_{O} \rightarrow 1^{+}} \bar{K}=+\infty$.

Proof: Since (39) is satisfied for each $\beta_{\mathrm{O}} \in\left(1, \beta_{\mathrm{O}}^{*}\right]$, we see from the proof of Theorem 2 that the delay bound of the closed-loop system can be solved from (25). In view of (40) and (41), we have

$$
\begin{aligned}
\lim _{\beta_{\mathrm{O}} \rightarrow 1^{+}} \frac{\beta_{\mathrm{O}}^{2}\left(1-\gamma^{*}\left(\beta_{\mathrm{O}}\right)\right)^{n-1} \gamma^{*}\left(\beta_{\mathrm{O}}\right)}{\left(\beta_{\mathrm{O}}-\left(1-\gamma^{*}\left(\beta_{\mathrm{O}}\right)\right)^{n}\right)^{3}} & =\lim _{\substack{\beta_{\mathrm{O}} \rightarrow 1^{+} \\
\gamma^{*}\left(\beta_{\mathrm{O}}\right) \rightarrow 0^{+}}} \frac{\gamma^{*}\left(\beta_{\mathrm{O}}\right)}{\left(\beta_{\mathrm{O}}-\left(1-\gamma^{*}\left(\beta_{\mathrm{O}}\right)\right)^{n}\right)^{3}} \\
& =\lim _{\substack{\beta_{\mathrm{O}} \rightarrow 1^{+} \\
\gamma^{*}\left(\beta_{\mathrm{O}}\right) \rightarrow 0^{+}}} \frac{1}{\left(\frac{\beta_{\mathrm{O}}-1}{\left(\gamma^{*}\left(\beta_{\mathrm{O}}\right)\right)^{\frac{1}{3}}}-\sum_{i=1}^{n}\left(\begin{array}{c}
n \\
i
\end{array}\right)(-1)^{i}\left(\gamma^{*}\left(\beta_{\mathrm{O}}\right)\right)^{i-\frac{1}{3}}\right)^{3}} \\
= & +\infty,
\end{aligned}
$$

where $\left(\begin{array}{c}n \\ i\end{array}\right)=\frac{n !}{i !(n-i) !}$.

Then, according to (25), we have

$$
\lim _{\beta_{\mathrm{O}} \rightarrow 1^{+}} 6 \bar{K}^{2}\left(\frac{\beta_{\mathrm{O}}}{\left(1-\gamma^{*}\right)^{n-1}}\right)^{2 \bar{K}}=+\infty .
$$

We next show that (42) implies $\lim _{\beta_{\mathrm{O}} \rightarrow 1^{+}} \bar{K}=+\infty$ by contradiction. Suppose $\bar{K} \leq \widetilde{K}$ for any $\beta_{\mathrm{O}} \in$ $\left(1, \beta_{\mathrm{O}}^{*}\right]$. Then, in view of $(40), \gamma^{*}\left(\beta_{\mathrm{O}}\right) \leq \overline{\gamma^{*}}$ for any $\beta_{\mathrm{O}} \in\left(1, \beta_{\mathrm{O}}^{*}\right]$, where $\overline{\gamma^{*}}$ is some positive constant. Thus, we have

$$
6 \bar{K}^{2}\left(\frac{\beta_{\mathrm{O}}}{\left(1-\gamma^{*}\right)^{n-1}}\right)^{2 \bar{K}} \leq 6 \widetilde{K}^{2}\left(\frac{\beta_{\mathrm{O}}^{*}}{\left(1-\overline{\gamma^{*}}\right)^{n-1}}\right)^{2 \widetilde{K}}<+\infty, \forall \beta_{\mathrm{O}} \in\left(1, \beta_{\mathrm{O}}^{*}\right]
$$

which contradicts (42). This completes the proof.

Example 1 Consider system (1) with

$$
A=\left[\begin{array}{ll}
1 & 0 \\
0 & a
\end{array}\right], B=\left[\begin{array}{l}
1 \\
1
\end{array}\right], C=\left[\begin{array}{ll}
1 & 1
\end{array}\right],
$$

where $a \in(1,1.05]$. It can be readily observed that as $a \rightarrow 1^{+}$, the exponentially unstable eigenvalue of $A$ approaches the unit circle. Also, $(A, B)$ is controllable and $(A, C)$ is observable. We design the observer gain matrix $L$ in (20) as $L=-\left[\begin{array}{ll}-1 & a+2\end{array}\right]^{\mathrm{T}}$, which places the eigenvalues of $A+L C$ at $\{ \pm \sqrt{2-a}\}$, inside the unit circle. Then, by $(26)$, we compute

$$
Q=\frac{-1}{a^{2}-4 a+3}\left[\begin{array}{cc}
a^{2}+4 a+9 & 2 a+6 \\
2 a+6 & 6
\end{array}\right] .
$$

Let $\gamma^{*}=1-1 / a$, which satisfies $\gamma^{*} Q<I$ for each $a \in(1,1.05]$. Therefore, according to the proof of Theorem 2 , the delay bound of the system is given in (25). Considering that $\beta_{\mathrm{O}}=a^{2}$ and $\gamma^{*}=1-1 / a$, 
we rewrite $(25)$ as

$$
6 \bar{K}^{2} a^{6 \bar{K}}=\frac{a^{2}(a-1)}{\left(a^{2}-a^{-2}\right)^{3}},
$$

whose right-hand side approaches infinity as $a \rightarrow 1^{+}$. Hence, $\lim _{a \rightarrow 1^{+}} \bar{K}=+\infty$, namely, as the unstable eigenvalue of $A$ approaches the unit circle, the delay bound goes to infinity. Alternatively, we can reach the same conclusion by verifying (39), (40) and (41) in Theorem 3.

Example 2 Consider system (1) with

$$
A=\left[\begin{array}{cc}
0 & -a \\
a & 0
\end{array}\right], B=\left[\begin{array}{l}
1 \\
0
\end{array}\right], C=\left[\begin{array}{ll}
1 & 0
\end{array}\right],
$$

where $a \in(1,1.24]$. The eigenvalues of $A$ are located at $\{ \pm j a\}$, which implies that as $a \rightarrow 1^{+}$, all the exponentially unstable eigenvalues of $A$ approach the unit circle. Also, we can verify that $(A, B)$ is controllable and $(A, C)$ is observable. Let $L=-\left[\begin{array}{ll}0 & a+1 / 2 a\end{array}\right]^{\mathrm{T}}$, which places the eigenvalues of $A+L C$ at $\{ \pm j \sqrt{2} / 2\}$, inside the unit circle. Then, solving (26), we obtain

$$
Q=\frac{1}{3}\left[\begin{array}{cc}
4+\frac{1}{a^{2}} & 0 \\
0 & 4+4 a^{2}
\end{array}\right] .
$$

Let $\gamma^{*}=1-1 / a$, which satisfies $\gamma^{*} Q<I$ for each $a \in(1,1.24]$. By substituting $\beta_{\mathrm{O}}=a^{4}$ and $\gamma^{*}=1-1 / a$ in (25), we obtain

$$
6 \bar{K}^{2} a^{10 \bar{K}}=\frac{a^{6}(a-1)}{\left(a^{4}-a^{-2}\right)^{3}},
$$

whose right-hand side goes to infinity as $a \rightarrow 1^{+}$. Therefore, $\lim _{a \rightarrow 1^{+}} \bar{K}=+\infty$. Similarly as in Example 1 , we can also reach the same conclusion by verifying (39), (40) and (41) in Theorem 3.

\section{$5 \quad$ Numerical Examples}

\subsection{Example for Theorem 1}

Consider system (1) with

$$
A=\left[\begin{array}{ccccc}
0 & 1 & 0 & 0 & 0 \\
0 & 0 & 1 & 0 & 0 \\
0 & 0 & 0 & 1 & 0 \\
-1 & 2 \sqrt{2} & -4 & 2 \sqrt{2} & 0 \\
0 & 0 & 0 & 0 & 1.02
\end{array}\right], B=\left[\begin{array}{l}
0 \\
0 \\
0 \\
1 \\
1
\end{array}\right]
$$

It can be readily verified that $(A, B)$ is controllable with $\lambda(A)=\{\sqrt{2} / 2 \pm j \sqrt{2} / 2, \sqrt{2} / 2 \pm j \sqrt{2} / 2,1.02\}$, from which we can see that $A$ is exponentially unstable with no eigenvalues inside the unit circle. Also, we compute $\beta=\operatorname{det}^{2}(A)=1.0404$. By solving (19), we obtain the delay bound as $K=2.7394$. Considering that $K \in \mathbb{N} \backslash\{0\}$, we set $K=2$. We can also determine the value of $\gamma^{*}$ to be $\gamma^{*}=1-\beta^{-\frac{1}{5}}=0.0079$. For the simulation purpose, we choose $\gamma=\gamma^{*}$ and the initial conditions to be $x(k)=\left[\begin{array}{lllll}1 & -1 & 0 & -1 & 1\end{array}\right]^{\mathrm{T}}$, $k=\{-2,-1,0\}$. We also choose two different $\phi(k)$ functions as follows. In the first case, $\phi(k)=k-2$, that is, a constant delay $\kappa=2$ is considered. In the second case,

$$
\phi(k)= \begin{cases}k, & \text { if } k=3 l+1, \\ k-2, & \text { if } k=3 l+2, \\ k-1, & \text { if } k=3 l, \quad l \in \mathbb{N},\end{cases}
$$

from which it can be verified that $\phi^{-1}(k)$ exists and is exactly known for $k \in \mathbb{N}$.

Simulation is run for both the constant delay case and the time-varying delay case. Shown in Figs. 1 and 2 are state response and control input of the system for the two delay cases. We can see that the asymptotic stability of the system is achieved in both cases. 

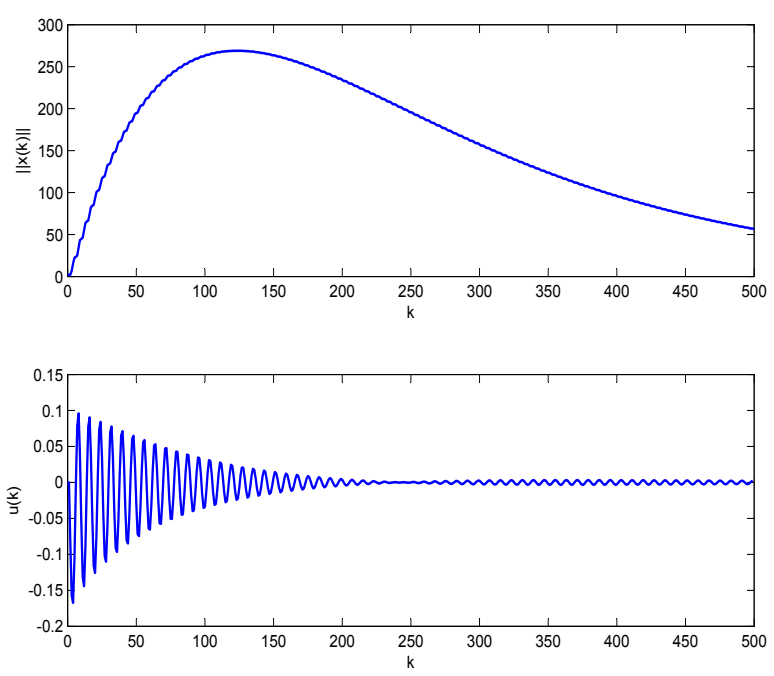

Figure 1: State response and control input of the system under the truncated predictor feedback law (8): the case of constant delay.
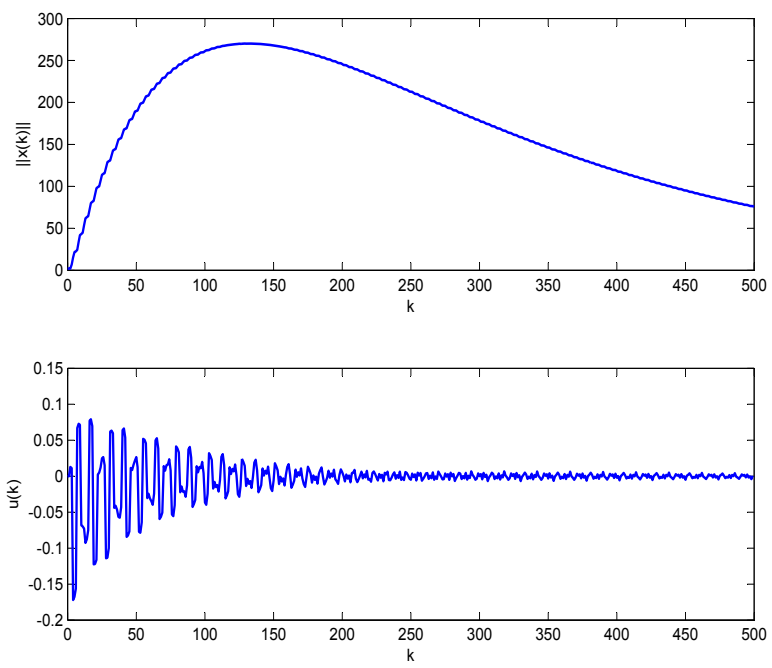

Figure 2: State response and control input of the system under the truncated predictor feedback law (8): the case of time-varying delay.

\subsection{Example for Theorem 2}

Consider system (1) with $(A, B)$ given by (43) and $C=\left[\begin{array}{lllll}1 & 1 & 1 & 1 & 1\end{array}\right]$. It can be readily verified

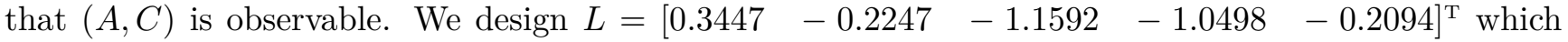
places the eigenvalues of $A+L C$ at $\{ \pm 0.05, \pm 0.1,0.95\}$, all of which are inside the unit circle. By (26) and $\gamma^{*} Q<I$, we set $\gamma^{*}=0.005$. Then, with the help of (25), we compute $\bar{K}=1.623$. For the simulation purpose, we set $\gamma=\gamma^{*}$ and $\kappa(k)=K=1$. Also, the system initial conditions are chosen to be $x(k)=-\hat{x}(k)=\left[\begin{array}{lllll}1 & -1 & 0 & -1 & 1\end{array}\right]^{\mathrm{T}}, k=\{-1,0\}$. Shown in Fig. 3 are the state response and control input of the system under truncated predictor output feedback law (20). 

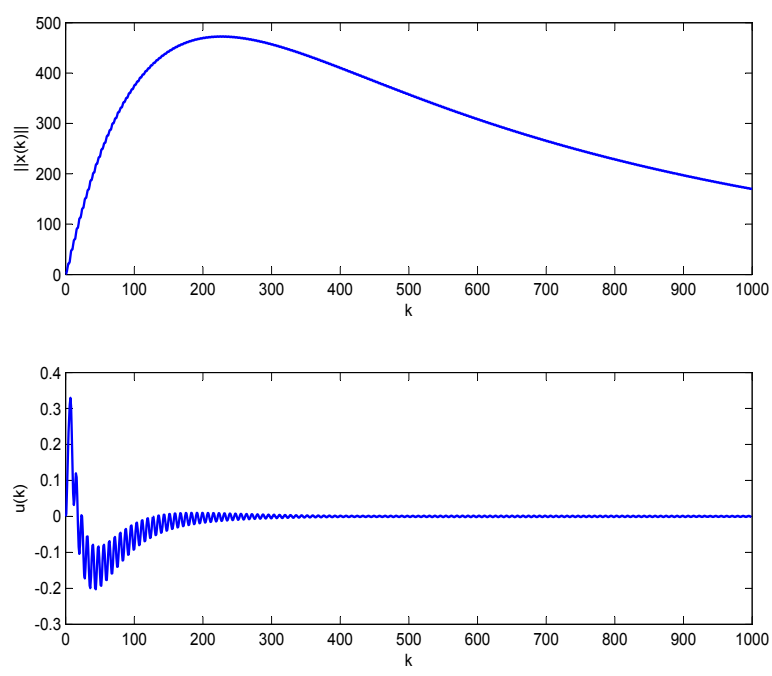

Figure 3: State response and control input of the system under the truncated predictor output feedback law (20).

\section{Conclusions}

In this paper, we considered the problem of stabilizing exponentially unstable discrete-time linear systems with time-varying input delay by using truncated predictor feedback, of both the state feedback type and the output feedback type. The feedback gain matrices in the truncated predictor feedback laws are constructed from the solution of a parameterized Lyapunov equation. Stability analysis leads to a bound on the delay and a range of the values of the parameter for which the closed-loop system is asymptotically stable as long as the delay is within the bound. The results are shown to extend the existing results on the stabilization by truncated predictor feedback for systems that are not exponentially unstable.

\section{References}

[1] Z. Artstein, "Linear systems with delayed controls: a reduction," IEEE Transactions on Automatic Control, Vol. 27, No. 4, pp. 869-879, 1982.

[2] R. Bellman and K. L. Cooke, Differential-Difference Equations, New York: Academic, 1963.

[3] J. Chen and A. Latchman, "Frequency sweeping tests for stability independent of delay," IEEE Transactions of Automatic Control, Vol. 40, No. 9, pp. 1640-1645, 1995.

[4] S. Elaydi and S. Zhang, "Stability and periodicity of difference equations with finite delay," Funkcialaj Ekvacioj, Vol. 37, No. 3, pp. 401-413, 1994.

[5] H. Fang and Z. Lin, "A further result on global stabilization of oscillators with bounded delayed input," IEEE Transactions on Automatic Control, Vol. 51, No. 1, pp. 121-128, 2006.

[6] H. Gao and T. Chen, "New results on stability of discrete-time systems with time-varying state delay," IEEE Transactions on Automatic Control, Vol. 52, No. 2, pp. 328-334, 2007.

[7] H. Gao, J. Lam, C. Wang and Y. Wang, "Delay-dependent output-feedback stabilisation of discretetime systems with time-varying state delay," IEE Proceedings-Control Theory and Applications, Vol. 151, No. 6, pp. 691-698, 2004. 
[8] K. Gu, V. L. Kharitonov, and J. Chen, Stability of Time-Delay Systems, Boston, MA: Birkhäuser, 2003.

[9] K. Gu, "An integral inequality in the stability problem of time-delay systems," Proc. 39th IEEE Conference on Decision and Control, Sydney, Australia, pp. 2850-2810, 2000.

[10] M. Krstic, "Compensation of infinite-dimensional actuator and sensor dynamics," IEEE Control Systems Magazine, Vol. 30, No. 1, pp. 22-41, 2010.

[11] M. Krstic, "Lyapunov stability of linear predictor feedback for time-varying input delay," IEEE Transactions on Automatic Control, Vol. 55, No. 1, pp. 554-559, 2010.

[12] Z. Lin, Low Gain Feedback, London. U.K.: Springer-Verlag, 1998.

[13] Z. Lin and H. Fang, "On asymptotic stabilizability of linear systems with delayed input," IEEE Transactions on Automatic Control, Vol. 52, No. 6, pp. 998-1013, 2007.

[14] Z. Lin, "On asymptotic stabilizability of discrete-time linear systems with delayed input," Communications in Information and Systems, Vol. 7, No. 3, pp. 227-264, 2007.

[15] F. Mazenc, S. Mondie and R. Francisco, "Global asymptotic stabilization of feedforward systems with delay in the input," IEEE Transactions on Automatic Control, Vol. 49, No. 5, pp. 844-850, 2004 .

[16] F. Mazenc, S. Mondie and S. I. Niculescu, "Global asymptotic stabilization for chain of integrators with a delay in the input," IEEE Transactions on Automatic Control, Vol. 48, No. 1, pp. 57-63, 2003.

[17] F. Mazenc, S. Mondie and S. I. Niculescu, "Global stabilization of oscillators with bounded delayed input," Systems $\&$ Control Letters, Vol. 53, No. 5, pp. 415-422, 2004.

[18] S.Y. Yoon, A. Parinya and Z. Lin, "An LMI approach to the control of exponentially unstable systems with input time delay," In Proc. of the IEEE Conference on Decision and Control, Florence, Italy, pp. 837-844, 2013.

[19] S.Y. Yoon and Z. Lin, "Truncated predictor feedback control for exponentially unstable linear systems with time-varying input delay," Systems $\&$ Control Letters, Vol. 62, No. 10, pp. 312-317, 2013.

[20] Q.C. Zhong, "On distributed delay in linear control laws - Part I: discrete-delay implementation," IEEE Transactions on Automatic Control, Vol. 49, No. 11, pp. 2074-2080, 2004.

[21] Q.C. Zhong, "On distributed delay in linear control laws - Part II: rational implementations inspired from the $\delta$-operator," IEEE Transactions on Automatic Control, Vol. 50, No.5, pp. 729-734, 2005.

[22] B. Zhou, G. Duan and Z. Lin, "A parametric Lyapunov equation approach to the design of low gain feedback," IEEE Transactions on Automatic Control, Vol. 53, No. 6, pp. 1548-1554, 2008.

[23] B. Zhou, Z. Lin and G. R. Duan, "A parametric Lyapunov equation approach to low gain feedback design for discrete-time systems," Automatica, Vol. 45, No. 1, pp. 238-244, 2009.

[24] B. Zhou and Z. Lin, "Parametric Lyapunov equation approach to stabilization of discrete-time systems with input delay and saturation," IEEE Transactions on Circuits and Systems I: Regular Papers, Vol. 58, No. 11, pp. 2741-2754, 2011.

[25] B. Zhou, Z. Lin and G. R. Duan, "Truncated predictor feedback for systems with long time-varying input delay," Automatica, Vol. 48, No. 10, pp. 2387-2399, 2012. 